\title{
Liposomes as a Tool to Study Lipid Peroxidation in Retina
}

\author{
Natalia Fagali and Angel Catalá
}

Additional information is available at the end of the chapter

http://dx.doi.org/10.5772/46074

\section{Introduction}

In living organisms, the oxidative stress is associated with several physio- pathological affections (e.g. atherosclerosis, cancer, aging, neurodegenerative diseases). The oxidative stress is generally initiated by generation of reactive oxygen (ROS) and nitrogen species (RNS) (Halliwell \& Gutteridge, 1990). ROS are continuously formed during cellular metabolism and are removed by antioxidants defences. ROS from endogenous and exogenous sources results in continuous and accumulative oxidative damage to cellular components and alters many cellular functions. The most vulnerable molecules to oxidative damage are proteins, lipids and DNA (Kohen \& Nyska, 2002; Catalá, 2009, 2011a, 2011b).

In mammalian retina, free radicals and lipoperoxides seem to play important roles in the evolution of different retinopathies including glaucoma, cataractogenesis, diabetic retinopathy, ocular inflammation and retinal degeneration (Ueda et al., 1996; De La Paz \& Anderson, 1992). Because of free radicals production induces the lipid peroxyl radical formation, known as secondary free radicals products; this chain reaction of lipid peroxidation can damage the retina, especially the membranes that play important roles in visual function (Catalá, 2006). The retina is the neurosensorial tissue of the eye. It is very rich in membranes and therefore in polyunsaturated fatty acids (PUFAs) such as docosahexenoic acid (22:6 n-3), that are quite vulnerable to lipid peroxidation. Also, the human retina is a well oxygenated tissue. High-energy short-wavelength visible light promotes the formation of ROS which can initiate lipid peroxidation in the macula and elsewhere. The macular carotenoids are thought to combat light-induced damage mediated by ROS by absorbing the most damaging incoming wavelengths of light prior to the formation of ROS and by chemically quenching ROS once they are formed.

Although peroxidation in model membranes may be very different from peroxidation in biological membranes, the results obtained in model membranes may be used to progress 
our understanding of subjects that cannot be studied in biological membranes. Nevertheless, in spite of the relative simplicity of peroxidation of liposomal lipids model, these reactions are still relatively complex because they depend in a complex fashion on liposome type, reaction initiator and reaction medium (Fagali \& Catalá, 2009). This complexity is the most likely cause of the apparent contradictions of literature results.

Biological membranes are complex systems. In view of this complexity and in order to avoid collateral effects that may arise during lipid peroxidation process of whole retinal membranes, we have attempted to gain understanding of the mechanisms responsible for peroxidation in a simple model system, made by dispersing retinal lipids in the form of liposomes.

This chapter describes a very useful method to prepare liposomes with natural phospholipids and the necessary methodology to follow the lipid peroxidation of these liposomes.

\section{Materials and methods}

\subsection{Materials}

Chloroform, methanol, trizma base, butylated hydroxytoluene (BHT), $\mathrm{NaCl}, \mathrm{FeSO}_{4}$ heptahydrate and 2-thiobarbituric acid (TBA) were purchased from Sigma Chemical Co. Suitable plastic lab ware was used throughout this study to avoid effects of adventitious metals. Other reagents were of the highest quality commercially available. All solutions were prepared using distilled water treated with a Millipore Q system.

\subsection{Isolation of bovine retina}

Eyes were enucleated at slaughter (Frigorífico Gorina), transported in ice to laboratory where retinas were taken out within 1-2 h. Under red light and with all tubes and solutions in ice buckets, corneas were excised; lenses and vitreous were subsequently removed. Eye cups were inverted and retinas were carefully peeled from the eyes. Retinas were briefly homogenized in $0.15 \mathrm{M} \mathrm{NaCl}\left(1 \mathrm{ml} /\right.$ retina) $120 \mathrm{~s}$ (20on-20off) at $4{ }^{\circ} \mathrm{C}$ in an Ultraturrax X25 homogenizer at $7000 \mathrm{rpm}$.

\subsection{Lipid extraction}

Total lipids were extracted from retinal homogenates with chloroform/methanol $(2: 1 \mathrm{v} / \mathrm{v})$ (Folch et al, 1957) at $4{ }^{\circ} \mathrm{C}$ (sample:Folch $\left.=1: 5\right)$. A volume of water corresponding to $20 \%$ of total volume was added. This mixture was shaken and kept in rest in cold to allow phases separation. Chloroformic phase was kept at $-22{ }^{\circ} \mathrm{C}$.

\subsection{Preparation of liposomes made of retinal lipids}

Total lipids obtained from retinal homogenates dissolved in chloroform were evaporated under nitrogen until constant weight and submitted to vacuum to remove traces of 
chloroform. Resultant films were dispersed at room temperature in a saline solution $(0.15 \mathrm{M}$ $\mathrm{NaCl}$ ). Dispersed lipids were mixed to homogeneity using a vortex-mixer to obtain nonsonicated liposomes (NSL). Sonicated liposomes (SL) were prepared by sonication of NSL under nitrogen and ice cooling (Huang, 1969), using a Sonics vibra cell, probe-sonicator Model VCX $750(750 \mathrm{~W}, 20 \mathrm{kHz})$ at $75 \%$ of maximal output. Preparation of liposomes required about $2.5 \mathrm{~min}$ of sonication to reach apparently minimal optical density values.

\subsection{Determination of liposomes size by Dynamic Light-Scattering (DLS)}

The time correlation $G(q, t)$ of the light-scattering intensity was measured at $90^{\circ}$ with a goniometer, ALV/CGS-5022F, with a multiple- $\tau$ digital correlator, ALV-5000/EPP, covering a $10^{-6}-10^{3} \mathrm{~s}$ time range. The light source was a helium/neon laser with a wavelength of $633 \mathrm{~nm}$ operating at $22 \mathrm{~mW}$. Each correlation function was analyzed by the well known cumulant fit yielding the apparent mean diffusion coefficient and the distribution $\delta D$ of this value (Koppel, 1972). The measurements were carried out with $80 \mu \mathrm{l}$ of SL and NSL (lipid concentration= $2 \mathrm{mg} / \mathrm{ml}$ ) in water, $0.15 \mathrm{M} \mathrm{NaCl}$ and Tris- $\mathrm{HCl}$ buffer $20 \mathrm{mM}$ (final volume $=2 \mathrm{~mL}$ ).

\subsection{Measurements of lipid peroxidation by detection of conjugated dienes and trienes production}

In order to determine conjugated dienes and trienes production, absorption spectra were recorded by means of a Shimadzu UV-1800 spectrophotometer, in the range 200 to $300 \mathrm{~nm}$, at $22{ }^{\circ} \mathrm{C}$, with $1 \mathrm{~cm}$ path length quartz cell. Liposomes made of retinal lipids $(80 \mu \mathrm{l}, 2 \mathrm{~g} / \mathrm{l}$ of lipids) were diluted to $2 \mathrm{ml}$ with water, $0.15 \mathrm{M} \mathrm{NaCl}$ or $20 \mathrm{mM}$ Tris- $\mathrm{HCl} \mathrm{pH} 7.4$, and oxidation was initiated by the addition of $\mathrm{FeSO}_{4}$ (final concentration $=25 \mu \mathrm{M}$ ). Lipid peroxidation was assessed continuously by measuring the increase in absorbance at $234 \mathrm{~nm}$ (formation of conjugated dienes) and $270 \mathrm{~nm}$ (formation of conjugated trienes) taken at 1 min intervals. Oxidation rates were determined as the slope of a regression line drawn through linear range of absorbance versus time curve. Lag times were determined as time corresponding to intersection of oxidation rate regression line with a regression line drawn through initial phase of oxidation (Sargis \& Subbaiah, 2003).

\subsection{Measurements of lipid peroxidation by detection of Thiobarbituric Reactive Substances (TBARS)}

During $\mathrm{Fe}^{2+}$ - initiated reactions, extent of liposomal lipid peroxidation was assessed using a TBA assay. In this procedure, $850 \mu \mathrm{L}$ of TBA $(0.375 \% \mathrm{w} / \mathrm{v}$ TBA, $0.25 \mathrm{~N} \mathrm{HCl})$ were added to aliquots of $150 \mu \mathrm{l}$ of reaction mixture containing BHT $(0.1 \% \mathrm{w} / \mathrm{v}$ in ethanol) to prevent possible peroxidation of liposomes during incubation. The aliquots were taken at different intervals of time. Samples were heated for $30 \mathrm{~min}$ at $75{ }^{\circ} \mathrm{C}$. Absorbance was measured at $532 \mathrm{~nm}$ for determination of aldehydic breakdown products of lipid peroxidation. 


\subsection{Preparation of Fatty Acids Methyl Esters (FAME)}

Lipids from retina, liposomes or liposomes exposed to peroxidation initiated by $\mathrm{Fe}^{2+}$, in absence or presence of BHT, were extracted according to the method of Folch et al (1957). A similar reaction mixture to that used in the analysis of conjugated dienes but scaled up 7.5 times was used to analyze the fatty acid composition of the samples

After one hour of incubation of liposomes with or without $\mathrm{Fe}^{2+}$ in the presence or absence of BHT, the samples were mixed with $15 \mathrm{ml}$ of chloroform:methanol $(2: 1 \mathrm{v} / \mathrm{v})$ containing $0.01 \%$ BHT to stop the reaction. The mixture was stirred, gassed with nitrogen and kept in refrigerator overnight to achieve separation of phases. The lower chloroform phase was filtered through paper filter containing anhydrous sodium sulphate. The solvent was evaporated to dryness under nitrogen. Dry lipids of retina and/or liposomes were transmethylated with $300 \mu \mathrm{l}$ of $1.3 \mathrm{M} \mathrm{BF}_{3}$ in methanol at $65^{\circ} \mathrm{C}$ during $180 \mathrm{~min}$. After incubation $1 \mathrm{ml}$ of $0.15 \mathrm{M} \mathrm{NaCl}$ was added and the fatty acid methyl esters were extracted with $1 \mathrm{ml}$ of hexane. This phase was injected onto the chromatograph.

\subsection{Gas chromatography - Mass spectrometry analyses}

GC-MS analyses were done using a Perkin Elmer Clarus 560D MS - gas chromatograph equipped with a mass selective detector with quadrupole analyzer and photomultiplier detector and a split/splitless injector. In the gas chromatographic system, a Elite 5MS (Perkin Elmer) capillary column ( $30 \mathrm{~m}, 0.25 \mathrm{~mm} \mathrm{ID}, 0.25 \mu \mathrm{m} \mathrm{df})$ was used. Column temperature was programmed from 130 to $250{ }^{\circ} \mathrm{C}$ at a rate of $5{ }^{\circ} \mathrm{C} / \mathrm{min}$ and $250{ }^{\circ} \mathrm{C}$ for $6 \mathrm{~min}$. Injector temperature was set to $260{ }^{\circ} \mathrm{C}$ and inlet temperature was kept at $250{ }^{\circ} \mathrm{C}$. Split injections were performed with a 10:1 split ratio. Helium carrier gas was used at a constant flow rate of 1 $\mathrm{ml} / \mathrm{min}$. In the mass spectrometer, electron ionization (EI+) mass spectra was recorded at 70 $\mathrm{eV}$ ionization energy, in full scan mode (50-400) unit mass range. The ionization source temperature was set at $180{ }^{\circ} \mathrm{C}$. The fatty acid composition of the lipid extracts was determined by comparing their methyl derivatives mass fragmentation patterns with those of mass spectra from the NIST databases.

\section{Results}

\subsection{Size of sonicated and non-sonicated liposomes made of retinal lipids in different aqueous media}

Average hydrodynamic radii of liposomes determined by DLS studies are presented in Table 1. We noted that NSL display a multimodal size distribution when analyzed by inverse Laplace transform (CONTIN), a result that is compatible with the high polydispersity index (PI > 0.4) from cumulants fit. Thus, hydrodynamic radii for NSL, at room temperature in different aqueous media, cover a broad range with intensity weighted maxima centered between 190 and $320 \mathrm{~nm}$. On the other hand, results for liposomes formed by sonication gave, through cumulant method, hydrodynamic radii in the order of 76.4-83.3 
$\mathrm{nm}$, showing as expected significant influence of sonication on size and distribution. It is clear that NSL possessed higher hydrodynamic radii than SL. Either NSL or SL in water were slightly smaller than that in $0.15 \mathrm{M} \mathrm{NaCl}$ and Tris-buffer.

\begin{tabular}{|c|c|c|c|}
\hline Type of liposome & Aqueous media & Hydrodynamic radii (nm) & Polydispersity index \\
\hline Sonicated & Water & 76.4 & 0.31 \\
\hline & $0.15 \mathrm{M} \mathrm{NaCl}$ & 83.3 & 0.27 \\
\hline & $20 \mathrm{mM}$ Tris-HCl & 83.3 & 0.27 \\
\hline Non-sonicated & Water & $190-225$ & 0.44 \\
\hline & $0.15 \mathrm{M} \mathrm{NaCl}$ & $260-320$ & 0.45 \\
\hline & $20 \mathrm{mM}$ Tris-HCl & $200-240$ & 0.43 \\
\hline
\end{tabular}

Table 1. Summary of values obtained by dynamic light scattering of SL and NSL made of retinal lipids in different aqueous media. Hydrodynamic radii values are the average of at least three representative determinations in each media.

\subsection{Evolution of UV spectra as a function of time for $\mathrm{Fe}^{2+}$ initiated lipid peroxidation of SL and NSL in different aqueous media}

Figure 1 shows evolution of UV spectra as a function of time, for $\mathrm{Fe}^{2+}$ initiated lipid peroxidation of SL and NSL, in different aqueous media. This figure showed increases in UV absorption with a maximum at $234 \mathrm{~nm}$ and at $270 \mathrm{~nm}$, due to conjugated dienes and trienes respectively, and a decrease of absorbance at 200-215 nm, due to loss of methylene interrupted double bonds (unoxidized lipids). When lipid peroxidation was carried out in water or $0.15 \mathrm{M} \mathrm{NaCl}$ decreases at $200-215 \mathrm{~nm}$ were more notorious than in reactions carried out in Tris-buffer.

\subsection{Conjugated dienes, trienes and TBARS are excellent markers of lipid peroxidation of liposomes made of retinal lipids}

Figure 2 shows changes in TBARS production and variation of absorbance at $234 \mathrm{~nm}$ and $270 \mathrm{~nm}$ as a function of time.

When SL were peroxidized in water (Figure 2A) a lag phase of $30 \mathrm{~min}$, followed by a fast rate, was observed in TBARS production. Absorbance final value at $532 \mathrm{~nm}$ reached was 0.24 . Increase of absorbance at $234 \mathrm{~nm}$ showed a small lag phase followed by a fast initial phase until $40 \mathrm{~min}$, since then speed of reaction became slighter. This behaviour was also observed in measured absorbance at $270 \mathrm{~nm}$, but all absorbance values were lower than that at $234 \mathrm{~nm}$ in the range of time studied.

Lipid peroxidation of SL in $0.15 \mathrm{M} \mathrm{NaCl}$ (Figure 2B). showed an immediate and fast production of TBARS without lag phase, reaching a final value (Abs $\approx 0.23$ ) similar to that obtained in water. The absorbance at $234 \mathrm{~nm}$ increased with an initial speed greater than 

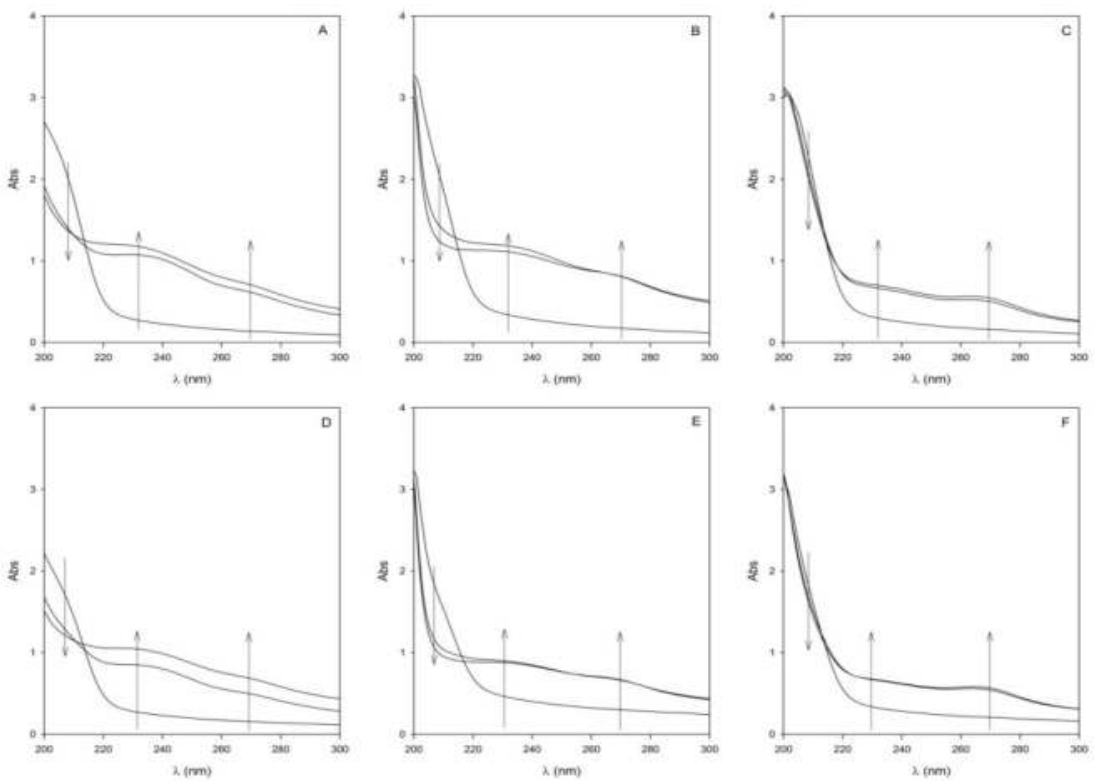

Figure 1. Time evolution ( 0,90 and $180 \mathrm{~min}$ ) of $\mathrm{UV}$ spectra of liposomes peroxidized with $\mathrm{Fe}^{2+}$ as an initiator of the reaction. SL in A) water, B) $0.15 \mathrm{M} \mathrm{NaCl}$, C) buffer Tris. NSL in D) water and) $0.15 \mathrm{M}$ $\mathrm{NaCl}, \mathrm{F}$ ) buffer Tris
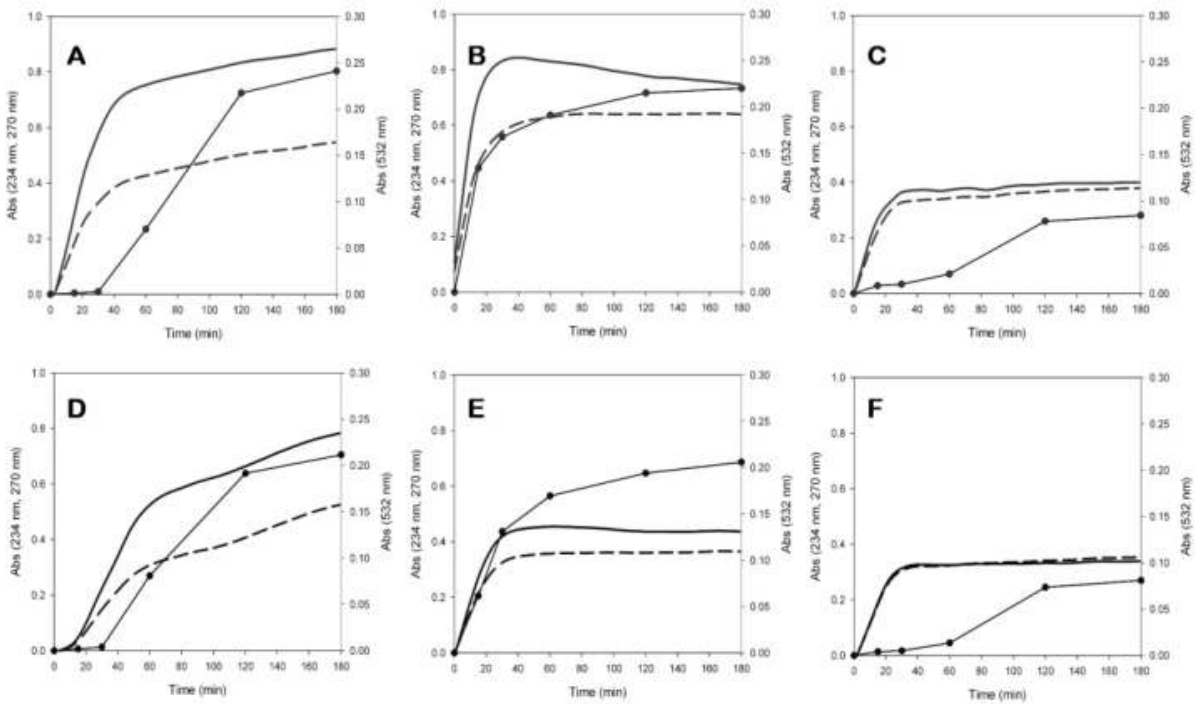

Figure 2. TBARS production (-•-) and variation of absorbance at $234 \mathrm{~nm}(-)$ and $270 \mathrm{~nm}(--)$ as a function of time, during $\mathrm{Fe}^{2+}$-catalyzed lipid peroxidation of SL (top) and NSL (bottom). TBARS were determined at $0,15,30,60,120$ and 180 min after addition of $\mathrm{Fe}^{2+}$. Aqueous media where reactions were carried out: A, D: water; B, E: $0.15 \mathrm{M} \mathrm{NaCl}$; C, F: 20 mM Tris-HCl pH 7.4. 
that observed in water, became the highest to 30 minutes and, then, diminished slowly. The absorbance at $270 \mathrm{~nm}$ increased with an initial speed greater than that observed in water, became the highest around the $30 \mathrm{~min}$ and then remained constant.

Lipid peroxidation of SL in Tris- buffer (Figure 2C) showed the largest lag phase and the lowest final value of absorbance $(\mathrm{Abs} \mathrm{f}=0.09)$ for TBARS formation. The initial speed of TBARS production was also the lowest. Initial speed of reaction observed, by increase of absorbance at $234 \mathrm{~nm}$, was lower than that measured on water and $0.15 \mathrm{M} \mathrm{NaCl}$. Absorbance reached the maximum at 30 minutes and then remained constant. Conjugated trienes production was very similar to that of conjugated dienes.

Lipid peroxidation of NSL in water (Figure 2D) showed a lag phase of $30 \mathrm{~min}$ for the TBARS production and a final value of absorbance of 0.21 . Changes of absorbance at $234 \mathrm{~nm}$ displayed a lag of $16 \mathrm{~min}$, increased quickly from this time to $60 \mathrm{~min}$ and since then continued increasing with lower speed. Values of absorbance at $270 \mathrm{~nm}$ were below than those observed at $234 \mathrm{~nm}$, although the behavior was similar.

Lipid peroxidation of NSL in $0.15 \mathrm{M} \mathrm{NaCl}$ (Figure 2E) showed an initial speed of TBARS production greater than that observed in water, but with a very similar final value (Abs= 0.21). Changes in absorbance at $270 \mathrm{~nm}$ and $234 \mathrm{~nm}$ showed greater initial speeds than the corresponding ones in water. These speeds stayed until 30 minutes and since then, absorbance values did not change. Final values of absorbance in $0.15 \mathrm{M} \mathrm{NaCl}$ were smaller than the water ones.

Lipid peroxidation of NSL in Tris-buffer (Figure 2F) showed the greatest lag phase (60 min) in TBARS production and the smallest initial reaction rate. The final value was 0.08 , a result much smaller than those obtained in water and $0.15 \mathrm{M} \mathrm{NaCl}$. Values of change of absorbance determined at $270 \mathrm{~nm}$ and $234 \mathrm{~nm}$ were practically the same. Initial speeds were similar to those obtained in water and slower to those observed in $0.15 \mathrm{M} \mathrm{NaCl}$. The reached final values were below to those obtained in water and $0.15 \mathrm{M} \mathrm{NaCl}$.

SL were more susceptible to lipid peroxidation than NSL both in water as in $0.15 \mathrm{M} \mathrm{NaCl}$. Nevertheless, both types of liposomes were equally peroxidized in Tris-buffer.

\subsection{Fatty acid composition of retinal lipids and liposomes made of these retinal lipids}

Figure 3 shows the fatty acid composition (area \%) of retinal lipids and of liposomes made of these retinal lipids (SL-Fe, control). This table also compares fatty acid profiles of control with liposomes incubated with $\mathrm{Fe}^{2+}$ for $1 \mathrm{~h}$, in absence and in presence of BHT. Retinal lipids show a high percent $(25.8 \pm 0.6 \%$ ) of docosahexaenoic acid (22:6n-3), characteristic of this tissue. The retina has approximately 40 percent of PUFAs and 60 percent of saturated and monounsaturated fatty acids. SL prepared with these lipids show a decrease of 22:6 n-3. The PUFAs diminished significantly after incubation with $\mathrm{Fe}^{2+}$. This produce a relative increase of saturated and monounsaturated fatty acids. $5 \mu \mathrm{M}$ BHT protected PUFAs avoiding lipid peroxidation effects and the fatty acid profile there was not significant differences with control. 


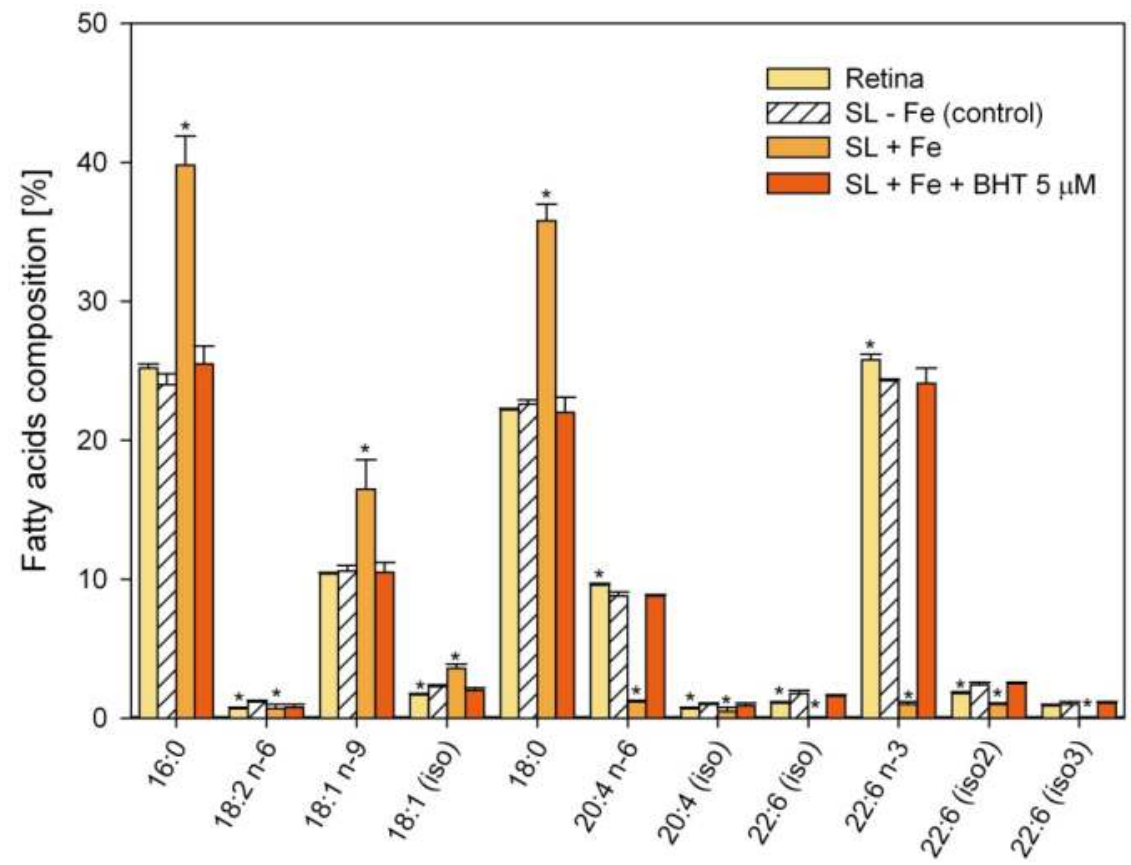

Figure 3. Fatty acid composition (area \%) of retinal lipids, liposomes made of retinal lipids (SL -Fe, control), liposomes incubated with $\mathrm{Fe}^{2+}(\mathrm{SL}+\mathrm{Fe})$ and liposomes incubated with $\mathrm{Fe}^{2+}$ in the presence of BHT. Results are expressed as $\tilde{x} \pm S D . \tilde{x}$ : Average of area \% of 3 assays, SD: standard deviation. Significant differences analyzed by ANOVA with control are marked with $\left(^{*}\right)$.

\section{Conclusion}

In summary, the presented results are indicative that liposomes made of retinal lipids by their structural similarities with the biomembranes constitute a very useful analytical system and can mimic the cellular membranes, providing additional information to that obtained with the whole retina. In addition, SL prepared with phospholipids obtained from selected tissues should be used in order to measure lipid peroxidation and the effect of different antioxidants. Additionally, we presented some simple techniques of many possibles that can be applied to study the lipid peroxidation process, different reaction initiators and the antioxidant effect of new compounds.

\section{Abbreviations}

16:0: palmitic acid, 18:1 n-9: oleic acid, 18:2 n-6: linoleic acid, 20:4 n-6: arachidonic acid, 22:6 n3: docosahexaenoic acid, BHT: butylated hydroxitoluene, GC-MS: gas chromatography-mass spectrometry, PUFAs: polyunsaturated fatty acids, TBARS: thiobarbituric acid reactive substances, RNS: reactive nitrogen species, ROS: reactive oxygen species, SL: sonicated liposomes. 


\section{Author details}

Natalia Fagali* and Angel Catalá

Instituto de Investigaciones Fisicoquímicas Teóricas y Aplicadas,

(INIFTA-CCT La Plata-CONICET), Facultad de Ciencias Exactas,

Universidad Nacional de La Plata. Casilla de Correo 16, La Plata, Argentina

\section{Acknowledgement}

The financial support of Consejo Nacional de Investigaciones Científicas y Técnicas (CONICET), Argentina, Grant PIP-0157, is gratefully acknowledged. The authors thank at the Departamento de Física, Facultad de Ciencias Exactas, Universidad Nacional de La Plata, Argentina for providing light scattering measurements facilities. Special thanks to Prof. J.L. Alessandrini for fitting experimental data.

\section{References}

Halliwell, B. and Gutteridge, J.M. (1990). Role of free radicals and catalytic metal ions in humandisease: an overview, Methods Enzymol. 186, 1-85

Huang, C. (1969). Studies on phosphatidylcholine vesicles, formation and physical characteristics. Biochemistry 8, 344-352.

Kohen, R., Nyska, A. (2002). Oxidation of biological systems: oxidative stress phenomena, antioxidants, redox reactions, and methods for their quantification. Toxicol. Pathol. 30, 620-650.

Catalá, A. (2009). Lipid peroxidation of membrane phospholipids generates hydroxyalkenals and oxidized phospholipids active in physiological and/or pathological conditions, Chem Phys Lipids, 157, 1-11.

Catalá, A. (2011a). In: Catalá, A. (Ed.), Lipid Peroxidation: Biological Implications, Transworld Research Network Kerala, India, pp. 1-173.

Catalá, A. (2011b). Lipid peroxidation of membrane phospholipids in the vertebrate retina, Front. Biosci. (Schol Ed). 3, 52-60, Review.

Fagali, N., Catalá, A. (2009) $\mathrm{Fe}^{2+}$ and $\mathrm{Fe}^{3+}$ initiated peroxidation of sonicated and nonsonicated liposomes made of retinal lipids in different aqueous media, Chem Phys Lipids, 159, 88-94.

Folch, J., Lees, N., Sloane Stanley, G.A. (1957). A simple method for the isolation and purification of total lipids from animal tissues. J Biol Chem, 226, 497-509.

Koppel, D.E. (1972). Analysis ofmacromolecular polydispersity in intensity correlation spectroscopy: the methods of cumulants, J Chem Phys, 57, 4814-4820.

Sargis, R.M., Subbaiah, P.V. (2003). Trans unsaturated fatty acids are less oxidizable than cis unsaturated fatty acids and protect endogenous lipids from oxidation in lipoproteins and lipid bilayers. Biochemistry, 42, 11533-11543.

\footnotetext{
${ }^{*}$ Corresponding Author
} 
Ueda, T., Ueda, T., Armstrong, D. (1996). Preventive effect of natural and synthetic antioxidants on lipid peroxidation in the mammalian eye. Ophthalmic Res, 28, 184192. 